\title{
Téoros
}

Revue de recherche en tourisme

\section{Le tourisme, au risque du politique}

\section{Bruno Sarrasin}

Volume 23, numéro 1, printemps 2004

$\mathrm{Au}$ risque du politique

URI : https://id.erudit.org/iderudit/1071361ar

DOI : https://doi.org/10.7202/1071361ar

Aller au sommaire du numéro

Éditeur(s)

Université du Québec à Montréal

ISSN

0712-8657 (imprimé)

1923-2705 (numérique)

Découvrir la revue

Citer ce document

Sarrasin, B. (2004). Le tourisme, au risque du politique. Téoros, 23(1), 3-4. https://doi.org/10.7202/1071361ar d'utilisation que vous pouvez consulter en ligne.

https://apropos.erudit.org/fr/usagers/politique-dutilisation/ 


\section{Le tourisme, au risque du politique}

\section{Bruno Sarrasin}

e tourisme est une industrie fragile. La

-lemande touristique est vulnérable à une multitude de phénomènes économiques, politiques et sociaux difficiles à comprendre, sinon à prévoir. Que ceux-ci soient réels ou simplement anticipés, l'effet demeure le même : le touriste potentiel décidera de reporter ses vacances ou choisira une destination qu'il juge mieux adaptée à ses besoins. Les récentes conjonctures qui ont marqué les principaux pays émetteurs et récepteurs de touristes au cours des dernières années (attentats du 11 septembre 2001 et ceux qui ont suivi, crise du syndrome respiratoire aigu sévère, guerre en Irak, etc.) donnent l'impression que le monde amorce une période particulièrement violente de son histoire et obligent les acteurs de l'industrie à considérer avec encore plus d'acuité les facteurs qui influencent l'évolution de la demande, en particulier le risque. Cet élément n'est pourtant pas nouveau puisque voyager a toujours été « risqué »... il suffit de se rappeler que les pèlerinages (entreprise de haut risque s'il en est) réunissaient déjà plusieurs caractéristiques des voyages contemporains. Plus récemment, la force des événements qui ont marqué l'actualité nous a fait oublier que la violence politique fait malheureusement partie du quotidien de plusieurs pays et régions du monde et que les touristes n'ont pas été épargnés au cours des vingt dernières années. Est-il plus risqué de voyager aujourd'hui ? Quels sont les effets sur le tourisme de l'évolution récente des relations internationales et dans quelle mesure le tourisme contribue-t-il à les façonner? Que devons-nous comprendre du « risque politique » pour mieux adapter l'offre touristique à l'évolution de la demande ?

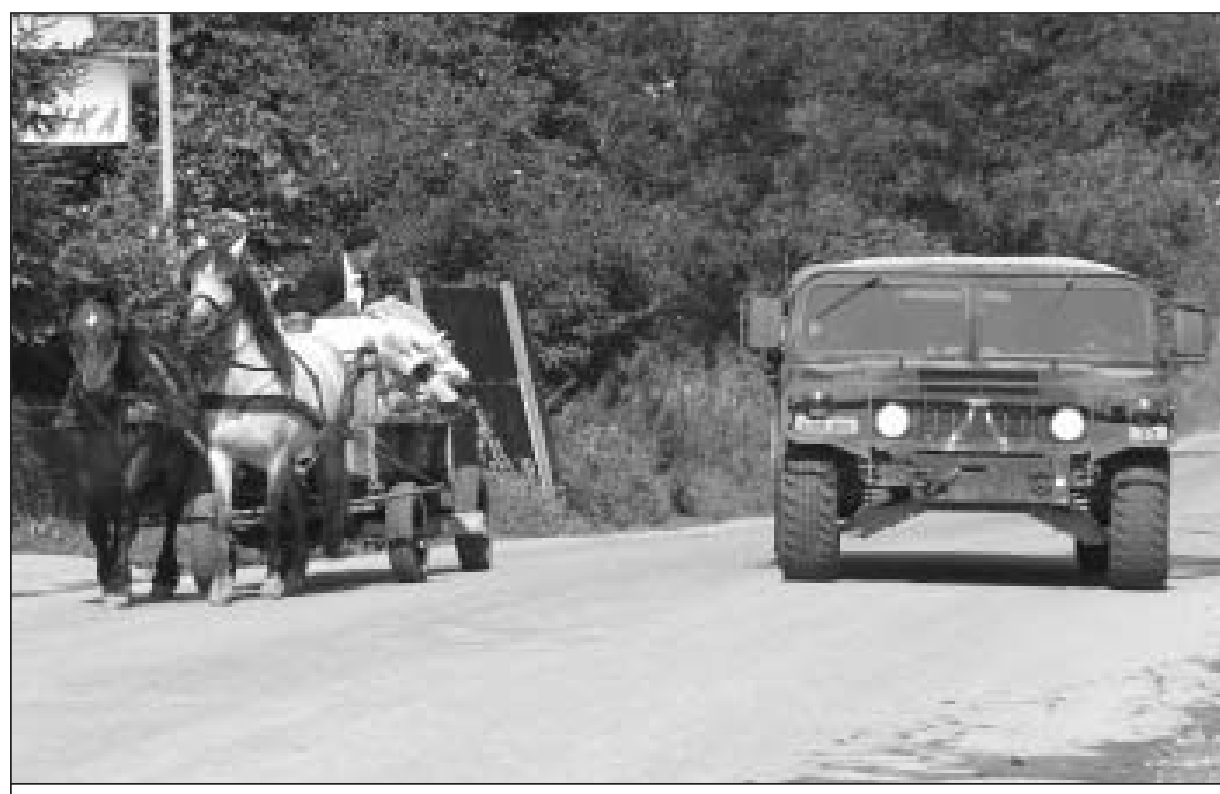

Véhicule militaire de la troupe américaine Task Force Eagle à Tuzia (Bosnie Herzégovine). Source: 0 rganisation du traité de l'Attantique $N$ ord (O TAN).

Bien que ce dossier n'ait pas la prétention de répondre à toutes les questions que se posent les acteurs de l'industrie, nous présentons une lecture transversale des enjeux que présente le « risque politique » en proposant huit articles aux approches disciplinaires diverses.

Le texte de Stéphane La Branche place, d'entrée de jeu, les enjeux fondamentaux du dossier. Si l'essor des attentats terroristes est dû en premier lieu à la fin de la guerre froide, celle-ci n'est pas suffisante pour expliquer pourquoi les attentats terroristes sont dirigés contre l'Occident et, plus généralement, quels sont les fondements de la violence politique. Cet article offre une réponse en proposant une analyse culturelle foucauldienne du système global. Il montre comment le terrorisme peut représenter une réaction à la violence politique, économique et identitaire issue de la mondialisation de la modernité occidentale. En d'autres termes, cette analyse permet de mieux comprendre les fondements de cette « nouvelle » instabilité politique. Nous savons combien la stabilité - pas seulement politique - représente une condition sine qua non au développement du tourisme. Le texte suivant (Sarrasin) explore les dimensions du «risque politique » pour mieux comprendre ses effets sur le tourisme. Il montre notamment que les représentations de la stabilité et de l'instabilité politique constituent une des caractéristiques fondamentales d'une destination touristique, dont l'importance dépasse souvent celle des attraits naturels et culturels. Il passe aussi en revue la dernière décennie en matière d'attentats visant des touristes. 


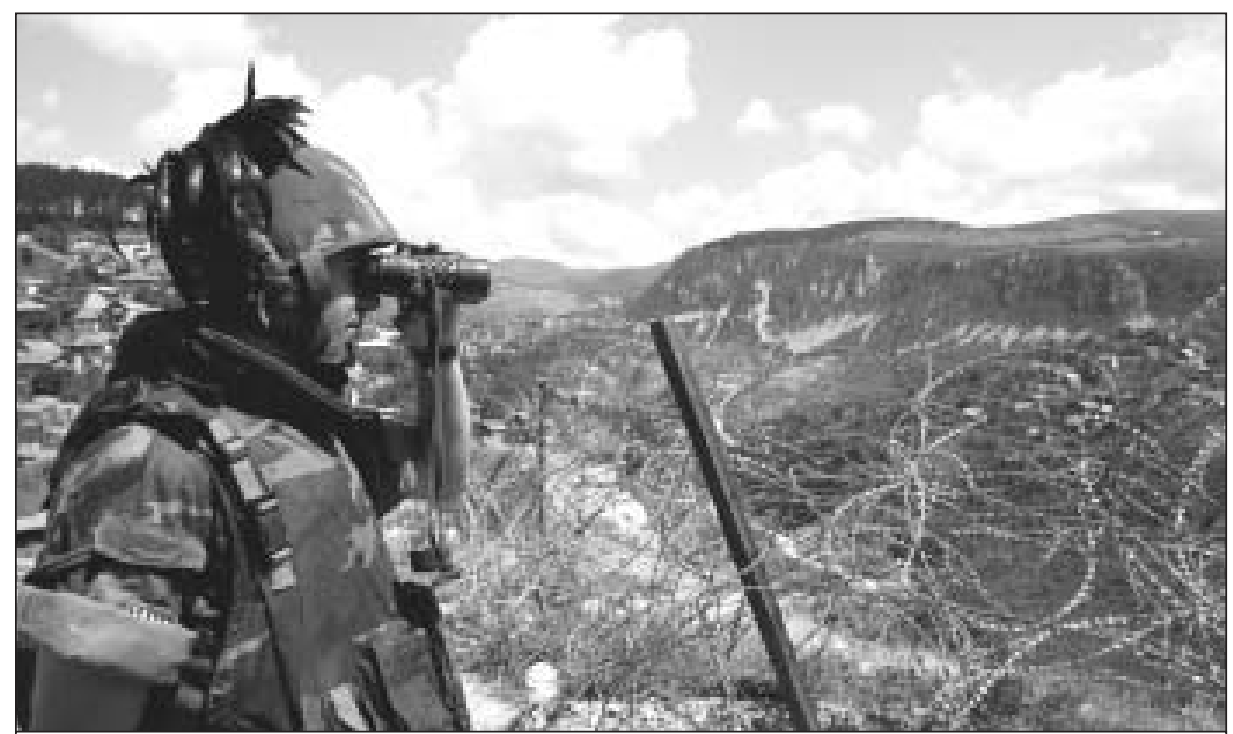

M ilitaire italien près de Sarajevo (Bosnie Herzégovine).

Source : O TAN

Les trois articles suivants placent les enjeux du dossier dans un contexte géographique précis. Mimoun Hillali analyse la relation entre pauvreté et violence, en prenant soin de décortiquer les liens entre les environnements économique et politique. Il montre, en utilisant des exemples issus du Maghreb, comment et pourquoi le tourisme (et les touristes) sont souvent utilisés comme vecteurs de déstabilisation et de médiatisation politique. L'article de Franck Michel utilise une approche similaire pour montrer que, dans plusieurs pays, le tourisme contribue à créer les propres conditions de son instabilité. Il suggère que, avec la mondialisation de l'insécurité, le touriste partage d'un coup le même destin que l'autochtone qui fait converger, du moins pour un moment, les conditions de vie de chacun. Cette lecture permet d'insister sur deux éléments fondamentaux pour comprendre l'environnement dans lequel se trouve aujourd'hui l'industrie touristique. D'abord, le tourisme et ses valeurs sous-jacentes (appropriation de l'espace et du temps à des fins ludiques, notamment) ne font pas l'unanimité. Le tourisme est un phénomène social, occidental, avant de devenir mondial. Ensuite, le tourisme n'est pas seulement victime de l'évolution des relations internationales et du « risque politique » qui en découle. Il participe activement à cette instabilité, générant des revenus, mais aussi des frustrations, et contribuant à sa façon au débat idéologique sous-jacent. En d'autres termes, « faire du tourisme » n'est pas une entreprise sans conséquences politique et sociale. Dans ce contexte, Julie Caron-Malenfant suggère que le poids de la couverture médiatique d'événements à caractère politique, impliquant ou non des touristes, doit être considéré comme une donnée importante par les planificateurs du tourisme, les intervenants de l'industrie et les différentes autorités publiques amenées à se pencher sur le phénomène touristique. Par son caractère polémique, le tourisme est une activité très exposée aux crises, entre autres en raison de la diversité de ses composantes, la mutation de ses formes et l'investissement émotionnel qu'il suppose.

Comment l'industrie et les consommateurs réagissent-ils à cet environnement en constante mutation ? Jacques Roy et Stéphanie Chrétien proposent une lecture sectorielle des effets de l'évolution récente du « risque politique » sur le tourisme. Le premier présente une analyse sectorielle du transport aérien qui a été plus que malmené par les conjonctures des trois dernières années. La réorganisation du secteur, particulièrement depuis les attentats du 11 septembre 2001, jumelée à un sentiment de risque accru face à ce mode de transport, pousse les consommateurs à redéfinir leurs comportements. C'est dans ce contexte que Jacques Roy présente les attentes des voyageurs d'affaires et la (ré)-émergence des transporteurs aériens à tarifs réduits. Le « risque politique » offrirait-il à l'industrie touristique de nouvelles occasions d'affaires ? Le second article propose une recherche exploratoire qui présente le processus actuel d'évaluation de projet que suivent les chaînes hôtelières internationales, en identifiant le plus exactement possible les sources de risques - pas seulement politiques - qui peuvent affecter la rentabilité. Stéphanie Chrétien conclut notamment que les chaînes hôtelières internationales n'adhèrent pas actuellement à un processus systématique d'analyse de risque. Contrairement à des domaines comme l'ingénierie et les technologies de l'information, la culture de gestion de projet, où s'insère l'analyse de risque, n'est pas encore reconnue dans le domaine du tourisme.

Enfin, l'article de Robert Lanquar propose à la fois une synthèse des enjeux de ce dossier et une réflexion sur l'avenir. Il analyse notamment la relation entre démocratie et tourisme, montrant par divers exemples que le premier est une condition essentielle au second. Le tourisme repose en effet sur le libéralisme économique (capitalisme) et politique (démocratie) pour se développer, mais ces conditions ne sont pas sans conséquence idéologique. Cet environnement, que les conjonctures politiques récentes ont mises à jour, placent l'industrie touristique à la croisée des chemins. Pour survivre à long terme, elle doit faire face à la réalité, c'est-à-dire aux risques à l'intérieur desquels les déplacements touristiques s'insèrent, mais aussi, génèrent. L'industrie doit aussi passer du mode « réactif » au mode « actif », d'une part, en cessant de nier l'existence des risques associés au voyage et, d'autre part, en intégrant cette dimension à la construction de l'offre. En attendant, on peut toujours se réjouir que les touristes oublient vite..., mais on ne peut pas construire l'avenir là-dessus !

Bruno Sarrasin est directeur de la revue Téoros et rédacteur invité pour ce dossier. 